\title{
Historia
}

\section{Hospital de San Lázaro de Granada: 470 años de historia, asistencia sanitaria y vida de los leprosos en el hospital ${ }^{*}$}

Saint Lazarus hospital of Granada: 470 years of history, health and life of hospital lepers

\author{
San Lazaro Hospital de Granada: \\ 470 anos de história, saúde e vida dos leprosos no hospital
}

\author{
Jessica Medina García ${ }^{1}$ \\ ${ }^{1}$ Diplomada en Enfermería, Masterando en la Universidad de Málaga: \\ Master Nuevas Tendencias en Investigación en Ciencias de la Salud.
}

Cómo citar este artículo en edición digital: Medina García, J. (2012) Hospital de San Lázaro de Granada: 470 años de historia, asistencia sanitaria y vida de los leprosos en el hospital.

16, 34. Disponible en: <http://dx.doi.org/10.7184/cuid.2012.34.05>

Correspondencia: Jessica Medina García. Calle Alcalá la Real no 100,

Edf. Jaima, Bloque 11, Piso 2c, CP 18013, Granada (Granada).Correo electrónico: jmg.enfermera@gmail.com Recibido 07/02/2012/ Aceptado: 10/07/2012

* III Simposio iberoamericano de historia de la enfermería, XII congreso nacional, VII internacional de historia de la enfermería y VII jornadas internacionales de cultura de los cuidados. Alicante 2011.

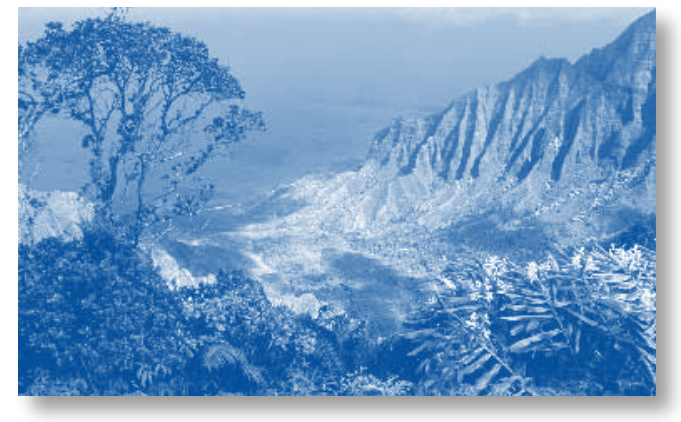

\section{ABSTRACT}

The Saint Lazarus Hospital was founded. Poverty and diseases were seen as a problem of public and social order and medical assistance as a state's task. However, the Church still kept an important role. The aim of this study is to present a general overview of the Saint Lazarus Hospital of Granada, from its beginning to its end, and to describe the lives of le- pers in the hospital, the organization of health care as well as the role of nurses. The material and methods used in this study include an exhaustive bibliography of electronic resources and books. The method consists of gathering documents published from 1996 to 2010, taking into account variables such as the opening date, its demolition, social and historical context of leprosy, medical assistance and the lives of patients. The main conclusions are: - The hospital was the only hospital founded in Granada in the sixteenth century which took care of lepers and aimed at reducing the spread of the disease. - The main figure of the medical assistance was the permanent doctor and the nurses.

Keywords: Saint Lazarus Hospital, Lepers, History, Nursing. 


\section{RESUMO}

O Hospital San Lazaro foi fundada por causa da pobreza e da doença nesta época começam a ser vistos como um problema de ordem pública e de assistência o trabalho social do Estado. No entanto, a Igreja continuará a manter um papel importante. O objetivo deste estudo é realizar uma visão geral do Hospital San Lazaro de Granada a partir do momento de abertura ao horário de fechamento, prescrever a vida dos leprosos no hospital e organização de saúde, descrevendo a figura de enfermagem. Os materiais e métodos utilizados têm sido uma busca exaustiva por meio de recursos eletrônicos e em formato papel. O método consiste em recolher documentos publicados de 1996 a 2010, tendo em conta variáveis como a abertura de datas e demolição do mesmo, contexto histórico e social da doença da lepra no momento, a organização dos cuidados e da vida dos leprosos em o hospital. As principais conclusões são que o San Lazaro Hospital foi o único hospital fundado em Granada, no século XVI e as funções de isolamento de cuidados, como a prevenção da hanseníase, a figura principal do atendimento médico foi a méica permanente e enfermeiros.

Palavras-chave: San Lazaro Hospital, Lepra, História, Enfermagem.

\section{RESUMEN}

El Hospital de San Lázaro fue fundado debido a que la pobreza y la enfermedad en esta época empiezan a ser consideradas como un problema de orden público y social, y la asistencia una labor del Estado. No obstante, la Iglesia seguirá manteniendo un papel importante. El objetivo del estudio es realizar una visión global del Hospital de San Lázaro de Granada desde el momento de su apertura hasta su cierre, prescribir la vida de los leprosos en el hospital y la organización asistencial, describiendo la figura de enfermería. El material y métodos utilizados han sido una exhaustiva búsqueda bibliográfica mediante recursos electrónicos y en formato papel. El método empleado consiste en recopilar documentación publicada desde el año 1996 al 2010, teniendo en cuenta variables como fechas de apertura y demolición del mismo, contexto histórico y social de la enfermedad de la lepra en la época, organización asistencial y vida de los leprosos en el hospital. Las conclusiones más destacadas son que el hospital fue el único fundado en Granada en el siglo XVI con función asistencial y de aislamiento como prevención de la lepra, la figura principal de la asistencia era el médico, dos enfermeros y dos enfermeras.

Palabras clave: Hospital San Lázaro, Leprosos, Historia, Enfermería.

\section{INTRODUCCIÓN}

La historia del Hospital de San Lázaro se extiende a lo largo de 470 años desde su instauración hasta su demolición. Por tanto, es un Hospital que en la actualidad se haya desaparecido, y sólo vivo en los libros y en el recuerdo de unos pocos. En 1502 fue fundado por los Reyes Católicos Don Fernando y Doña Isabel, para recluir en él a los enfermos de lepra (bacilo de Hanse) (Cambil Hernández, 2010), de ahí el nombre que recibió "Hospital San Lázaro”, ya que este es el patrón de los leprosos.

Tuvo varias ubicaciones, la primera fue en el Albaicín, en la casa de un noble árabe en la placeta de las Tomasas, pero al considerarse perjudicial para los vecinos (Barrios Rozúa, 2006), se trasladó cerca del río Genil (Cambil Hernández, 2010). Este lugar tampoco fue definitivo ya que en 1514 se volvió a trasladar, esta vez extramuros frente a la puerta de Elvi- 
ra, a un lado del barrio del Albayzín, cerca de la orilla izquierda del río Beiro. El padre de la Chica en el s. XVIII nos habla ya del Barrio de San Lázaro, en la actualidad más conocido como Plaza de Toros, haciendo referencia también a que el hospital estuvo en otros lugares.

Por tanto, el Hospital de San Lázaro quedo definitivamente situado a nivel urbano, en una zona extramuros, que posteriormente daría lugar al nombre del barrio adyacente, Barrio de San Lázaro (Cambil Hernández, 2010). Hay que destacar que el edificio en el que se instaló el hospital decisivamente no fue constituido para su fin, por eso no presenta la estructura de los lazaretos (Cambil Hernández, 2010).

El objetivo general consiste en realizar un estudio global del Hospital de San Lázaro de Granada desde el momento de su puesta en marcha hasta su cierre y el objetivo específico se centra en describir la vida de los leprosos en el hospital, la organización asistencial y, fundamentalmente, la figura de enfermería.

\section{MATERIAL Y MÉTODO}

Este trabajo se ha llevado a cabo desde un punto de vista histórico, social y sanitario, mediante una amplia búsqueda bibliográfica donde el material utilizado ha sido producto de una exhaustiva búsqueda bibliográfica mediante recursos electrónicos en bases de datos científicas de ciencias de la salud, considerando como principal fuente Pubmed y el catálogo de la Universidad de Granada. En el caso del formato papel, y consultas de tesis doctorales la búsqueda ha sido realizada en la Biblioteca Biosanitaria y en la Biblioteca de Filosofía y Letras de la misma universidad. El método ha consistido en recopilar documentación publicada desde el año 1996 al 2010, teniendo en cuenta variables como fechas de apertura y demolición del mismo, contexto histórico y social de la enfermedad de la lepra en la época, organización asistencial y vida de los leprosos en el hospital. En función de los objetivos se sitúan las aportaciones de los distintos autores, y se exponen las conclusiones más relevantes.

\section{RESULTADOS Y SU DISCUSIÓN}

El Hospital de San Lázaro era un sitio de reclusión de los afectados de lepra, al considerar la enfermedad como un mal contagioso e incurable, por lo que los poderes públicos intervenían directamente en su funcionamiento. En las constituciones del hospital se recogía que sus enfermos podían pedir limosna en los caminos, pero no en las ciudades, para evitar el contagio de la población. Lo curioso de esto es que las limosnas recogidas se distribuían dando un cuarto del total al mendigo y el resto para el hospital (Sánchez Dehesa García \& Universidad de Granada, 2003).

$\mathrm{Su}$ organización asistencial era bastante precaria, al médico titular era el responsable de la asistencia médica que se le daba al leproso, y era ayudado por un médico decano de la Facultad de Medicina de la Universidad de Granada, un cirujano titular, dos enfermeros y dos enfermeras (Sánchez Dehesa García \& Universidad de Granada, 2003).

El médico titular tenía como principal obligación asistir a los enfermos y dependientes del hospital, y reconocerlos para su acogida o alta en el hospital. El cirujano titular tenía funciones muy parecidas, ya que ambos visitaban diariamente a los enfermos, en compañía de los enfermeros y les prescribían los medicamentos necesarios. Además realizaba certificados de contagio o de curación a los enfermos que querían entrar o salir del hospital.

El médico decano confirmaba la dolencia que expresaba el médico y el cirujano en los certificados de los enfermos admitidos o 
despedidos, siendo su informe definitorio si ambos tenían diversidad de opiniones. Los dos enfermeros y las dos enfermeras eras los encargados de cumplir las prescripciones facultativas del médico y cirujano titular. Los practicantes con frecuencia alteraban el orden dietético o farmacológico que había sido prescrito por el médico, lo que hacía que la asistencia médica no fuera la correcta.

Los dos enfermeros tenían entre sus funciones atender a los enfermos varones ingresados, limpiar las enfermerías, asistir a los enfermos que estaban en la cama, llevándoles la comida y los medicamentos prescritos por los médicos, hacer la cama de los pacientes postrados, vigilar a los encamados, si había algún enfermo grave, se alternaban los dos enfermeros de día y de noche para atenderlo, y amortajaban cuando alguno moría.

Además debían pasar visita con el médico y llevar un cuaderno para anotar las prescripciones, entre las cuales se incluía la barbería. El enfermero también cuidaba de la botica, ocupándose de traer de fuera lo necesario y preparaba los ungüentos, refrescos, cocimientos, y remedios caseros fáciles. También uno de ellos tenía que acompañar al cirujano con otro cuaderno, en las visitas y curaciones, ayudándole en todo lo necesario, a curar las úlceras comunes de los leprosos, que por estar en cama no lo pudieran hacer ellos mismos. El segundo enfermero además de las tareas propias del enfermero también era barbero, tenia que afeitarles y cortarles el pelo a los leprosos. Las dos enfermeras sin embargo, atendían sólo a mujeres enfermas. Tenían como principal obligación el cuidado a las mujeres encamadas, servirles la comida, la limpieza de la enfermería, llevar a cabo las indicaciones que el médico mandaba, y cuidar del remiendo de toda la ropa de los enfermos.

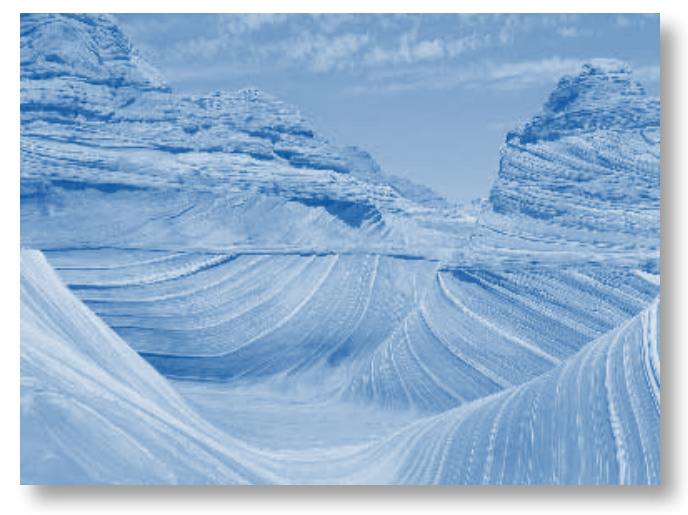

Estas funciones eran auxiliares, en comparación con las que hacían los enfermeros, e incluso cobraban menos (Sánchez Dehesa García \& Universidad de Granada, 2003).

Para la asistencia espiritual a los enfermos y el mantenimiento de la iglesia había un capellán, que era ayudado por el sacristán y el mayoral mampastor (Sánchez Dehesa García \& Universidad de Granada, 2003), cuyos quehaceres eran decir misa tanto los domingos como los días festivos y viernes de cada semana, además de enseñar la doctrina a los enfermos y ayudarles al buen morir (Cambil Hernández, 2010).

Para conocer mejor la vida de los leprosos en esta época hay que considerarlos dentro de un marco histórico y social. La lepra es una enfermedad infecciosa y crónica que llegó a Europa trasmitida por los ejércitos del Imperio Romano. A lo largo de los siglos VII y VIII, se produjo un recrudecimiento de la misma que provocó un internamiento de estos enfermos en hospitales y casas para tratar de evitar el contagio, llamadas leproserías. No obstante, la propagación de esta enfermedad en Europa vino determinada por dos hechos fundamentales: las Cruzadas y el Camino de Santiago. En los siglos XI y XII pasaron a tener un carácter endémico. A comienzos del siglo XV la lepra empezó a disminuir en Europa, aun que de forma más lenta en España y Portugal. Los fac- 


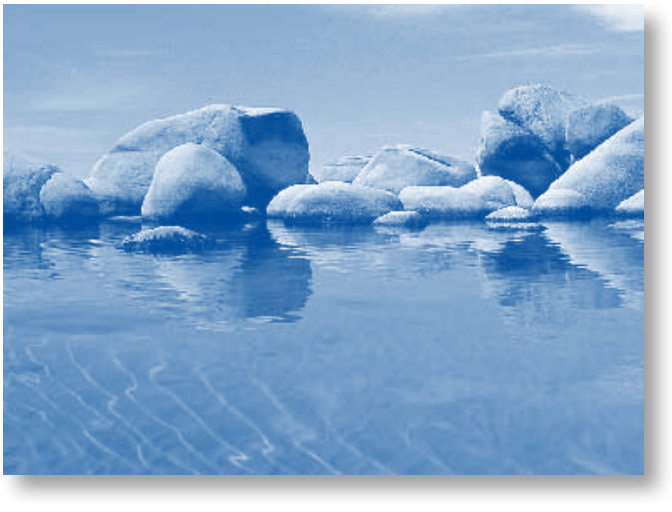

tores que influyeron en este descenso fueron la mejora de la política sanitaria, sobre todo en el aspecto de separación de leprosos del resto de la sociedad; los progresos de la higiene y mayor conocimiento sobre el diagnóstico y el contagio. Esta reducción se mantuvo en los siglos XVI, XVII y XVIII, que produjo cierta despreocupación por parte de las autoridades, lo cual produciría un recrudecimiento de la enfermedad a finales del siglo XIX.

También es de destacar la propagación de la lepra por Andalucía, Levante y Extremadura que tuvo lugar hasta el año 750 por los islámicos. En este caso, la propagación en los territorios del Al-Andalus fue tan austera porque los árabes no separaban a los leprosos del resto. Solo hacían tal cosa cuando los leprosos tenían un aspecto desagradable por sus deformaciones y mutilaciones. Por tanto, Al-Andalus padeció una endemia crónica de lepra (Sánchez Dehesa García \& Universidad de Granada, 2003).

Los leprosos estaban totalmente rechazados por la sociedad y hasta por sus propias familias, debido al miedo al contagio. Provocaban repulsión por las deformidades físicas que sufrían, las cuales contradecían los cánones de belleza que eran muy valorados desde el punto de vista social.

El estigma de la lepra se origina durante la Edad Media, debido al escaso desarrollo de la Medicina y por la influencia de explicaciones incoherentes de la Iglesia, el castigo divino. Los leprosos tenían la obligatoriedad de avisar de su presencia y de su existencia. Su vestimenta debía de ocultar las deformaciones físicas, con vestiduras largas hasta los pies, guantes y capuchón o sombrero para cubrirse la cabeza. Esta imposición fue más propia de la Edad Media, pero se mantuvo hasta el siglo XX (Sánchez Dehesa García \& Universidad de Granada, 2003).

La vida de los leprosos en el hospital mejoraba, sobre todo en el caso de los pobres. Encontraban en él su segundo hogar, dónde eran tratados como personas, al contrario que en la calle donde eran rechazados y eran considerados como los "apestados". Los leprosos podían realizar trabajos dentro del recinto hospitalario y llevar una vida social restringida (Sánchez Dehesa García \& Universidad de Granada, 2003).

El carácter de los enfermos de lepra, debido a la consideración social de su mal, los hacía ser personas introvertidas, tristes y con escasa valoración de sí misma (Sánchez Dehesa García \& Universidad de Granada, 2003). Sentían vergüenza de su propia enfermedad y lo ocultaba por miedo a ser recluidos.

Los factores que más influían en la actitud negativa frente a la lepra eran (García Monlleó \& Universidad de Granada, 1996):

- Influencia bíblica, ya que se consideraba como un castigo divino padecer la enfermedad.

- Evolución de la enfermedad a lo largo de la historia.

- Propagación en Asia, Europa y África.

- Iconografía social a través de las pinturas, escultural y literatura, que reforzó el prejuicio hacia la enfermedad. 
- El tener lepra originaba una "muerte social".

- Prejuicios milenarios.

- Asociación de la lepra con deformaciones físicas.

La lepra mutilaba a sus víctimas de una forma espantosa, gangrenando los miembros superiores e inferiores hasta que éstos se desprendían. Esto, unido al olor pestilente de las heridas, motivó el rechazo social de una de las enfermedades más temidas por aquellos entonces. En todo caso, la palabra lepra causaba horror, espanto, temor y rechazo social (Sanchez Garcia, 1996). En el 1792 el número de enfermos aumentó considerablemente, ya que en este año pasó a ser Hospital General de todos los reinos de España, acudiendo enfermos de todas partes de la península (Cambil Hernández, 2010), a excepción de Sevilla por tener un centro de similares características en la ciudad (Moreno Toral, 1997).

En 1839 el hospital paso a ser sustentado por la Administración General de Beneficencia de la Diputación Provincial de Granada que encargo la asistencia a los enfermos a las Hijas de la Caridad (Barrios Rozúa, 2006). Siguió funcionando como hospital de leprosos y otras enfermedades infecciosas.En 1937 la Comisión Gestora de Provincial acordó realizar importantes reformas por la necesidad evidente de la Guerra Civil. Pero aún así, el edificio quedó anticuado y se decidió trasladar sus servicios a otros centros más modernos (Barrios Rozúa, 2006). En 1971 fue desalojado (Barrios Rozúa, 2006) y en 1973 (Cambil Hernández, 2010) fue derribado. Hoy en día en su lugar se encuentra las oficinas de la Diputación y juzgados (Barrios Rozúa, 2006), el Centro de Salud Universitario La Caleta y El Centro Victoria Eugenia de Educación Infantil, primaria y de adultos (Cambil Hernández, 2010).

\section{CONCLUSIONES}

El hospital fue fundado porque la pobreza y la enfermedad eran consideradas como un problema de orden público y social, y la asistencia una labor del Estado, aun que la Iglesia siguió manteniendo un papel preponderante.

Fue el único hospital fundado en Granada en el siglo XVI con función asistencial y de aislamiento como prevención de la enfermedad.

Presenta diferencias con las demás instituciones del siglo XVI como son la especialización con respecto a sus enfermos atendiendo en su mayoría a leprosos.

En 1792 el hospital pasó a ser Hospital General del Reino de España, lo que aumento considerablemente el número de enfermos.

La figura principal de la asistencia es el medico titular, al que le ayudaba el medico cirujano y el medico decano. En el caso de los dos enfermeros y las dos enfermeras cumplían las prescripciones facultativas de los médicos. Las funciones de las enfermeras eran auxiliares, en comparación con las que hacían los enfermeros, e incluso cobraban menos.

Los practicantes con frecuencia alteraban el orden dietético o farmacológico que había sido prescrito por el médico, lo que hacía que la asistencia médica no fuera la correcta.

El tener lepra originaba una "muerte social", debido al rechazo por las deformidades físicas que sufrían, las cuales no correspondían a los cánones de belleza de la época, además muy valorados por la sociedad.

Los hospitales como San Lázaro y las leproserías eran el único asilo para estos enfermos, aquí al menos eran tratados como personas, convirtiéndose el hospital o leprosería como su segundo hogar, lo que influía positivamente en su enfermedad. 


\section{BIBLIOGRAFÍA}

- Barrios Rozúa, J. M. (2006) Guía de la Granada desaparecida (2da ed.). Editorial Comares, Granada..

- Cambil Hernández, Ma E. (2010) Los hospitales de Granada: (siglos XVI-XXI): tipología, catálogo e historia. Editorial Universidad de Granada, Granada.

- García Monlleó, F. J., \& Universidad de Granada. (1996) La asistencia hospitalaria en Granada: El Hospital de San Juan de Dios (1552-1835). Granada.
- Moreno Toral, E. (1997) Estudio social y farmacoterapéutico de la Lepra: El Hospital de San Lázaro de Sevilla (Siglo XIII-XIX). Diputación Provincial de Sevilla, Sevilla.

- Sánchez Dehesa García, R. M., \& Universidad de Granada. (2003). El Hospital de San Lázaro de Granada. 18001830, Granada.

- Sanchez Garcia, R. (1996) La lepra en España en la primera mitad del siglo XX. Universidad Complutense, Madrid.

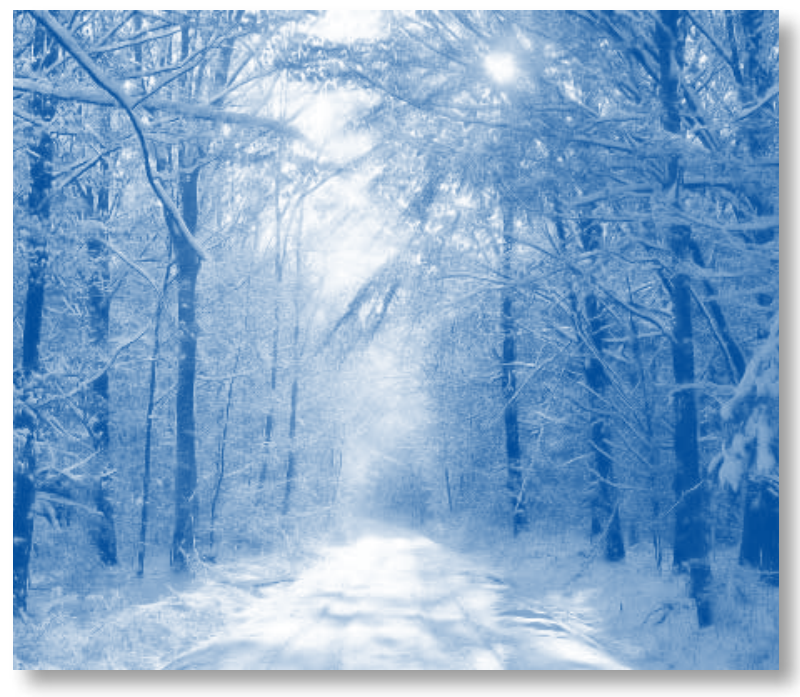

